\title{
Genome-wide discovery and characterization of long noncoding RNAs in African oil palm (Elaeis guineensis Jacq.)
}

\author{
Wei Xia ${ }^{\text {Equal first author, } 1}$, Yajing Dou ${ }^{\text {Equal first author, } 1}{ }^{,}$, Rui Liu ${ }^{2}$, Shufang Gong ${ }^{2}$, Dongyi Huang ${ }^{1}$, Haikuo Fan ${ }^{\text {Corresp., }}{ }^{2}$, Yong \\ Xiao ${ }^{\text {Corresp. } 2}$ \\ ${ }^{1}$ College of Tropical Crops, Hainan University, Haikou, China \\ 2 Coconut Research Institute, Chinese Academy of Tropical Agricultural Sciences, Wenchang, China \\ Corresponding Authors: Haikuo Fan, Yong Xiao \\ Email address: venheco@163.com, xiaoyong1980@catas.cn
}

Long noncoding RNAs (IncRNAs) are an important class of genes and play important roles in a range of biological processes. However, few reports have described the identification of IncRNAs in oil palm. In this study, we applied strand specific RNA-seq with rRNA removal to identify 1,363 IncRNAs from the equally mixed tissues of oil palm spear leaf and six different developmental stages of mesocarp (8-24 weeks). Based on strand specific RNAseq data and 18 released oil palm transcriptomes, we systematically characterized the expression patterns of IncRNA loci and their target genes. A total of 875 uniq target genes for natural antisense IncRNAs (NAT-IncRNA, 712), long intergenic noncoding RNAs (lincRNAs, 92), intronic-IncRNAs (33), and sense-IncRNAs (52) were predicted. A majority of IncRNA loci (77.8\% - 89.6\%) had low expression in 18 transcriptomes, while only 89 IncRNA loci had medium to high expression in at least one transcriptome. Coexpression analysis between IncRNAs and their target genes indicated that $6 \%$ of IncRNAs had expression patterns positively correlated with those of target genes. Based on single nucleotide polymorphism (SNP) markers derived from our previous research, 6,882 SNPs were detected for IncRNAs and 28 SNPs belonging to 21 IncRNAs were associated with the variation of fatty acid contents. Moreover, seven IncRNAs showed expression patterns positively correlated expression pattern with those of genes in de novo fatty acid synthesis pathways. Our study identified a collection of IncRNAs for oil palm and provided clues for further research into IncRNAs that may regulate mesocarp development and lipid metabolism. 
1 Genome-wide discovery and characterization of long noncoding RNAs in African oil palm (Elaeis guineensis

2 Jacq.)

3 Wei Xia*1, Yajing Dou*12, Rui Liu², Shufang Gong ${ }^{2}$, Dongyi Huang ${ }^{1}$, Haikuo Fan ${ }^{\& 23}$, Yong Xiao ${ }^{23}$

$4{ }^{1}$ Hainan Key Laboratory for Sustainable Utilization of Tropical Bioresources, College of Tropical Crops, Hainan

5 University, Haikou, 570228, P. R. China

$6 \quad{ }^{2}$ National Engineering Research Center of Coconut/Coconut Research Institute, Chinese Academy of Tropical

7 Agricultural Sciences, Wenchang, 571339, P. R. China

$8 \quad{ }^{3}$ Hainan Key Laboratory for Biosafety Monitoring and Molecular Breeding in Off-Season Reproduction Regions

$9 \quad *$ Equal contributor

$10 \&$ Corresponding author

11 Coconuts Research Institute, CATAS, Wenchang, Hainan 571339, P. R. China

12 Email address: venheco@163.com

13 Email address: xiaoyong1980@catas.cn

14

15

16

17

18

19

20

21

22

23

24

25

26

PeerJ reviewing PDF | (2019:07:39449:4:0:NEW 27 May 2020) 


\section{Abstract}

29 Long noncoding RNAs (lncRNAs) are an important class of genes and play important roles in a range of biological 30 processes. However, few reports have described the identification of IncRNAs in oil palm. In this study, we applied strand specific RNA-seq with rRNA removal to identify 1,363 lncRNAs from the equally mixed tissues of oil palm spear leaf and six different developmental stages of mesocarp (8-24 weeks). Based on strand specific RNA-seq data and 18 oil palm transcriptomes, we systematically characterized the expression patterns of lncRNA loci and their target genes. A total of 875 unique target genes for natural antisense lncRNAs (NAT-lncRNA, 712), long intergenic noncoding RNAs (lincRNAs, 92), intronic-lncRNAs (33), and sense-lncRNAs (52) were predicted. A majority of lncRNA loci $(77.8 \%$ - 89.6\%) had low expression in 18 transcriptomes, while only 89 lncRNA loci had medium to high expression in at least one transcriptome. Coexpression analysis between lncRNAs and their target genes indicated that $6 \%$ of IncRNAs had expression patterns positively correlated with those of target genes. Based on lncRNAs and 28 SNPs belonging to 21 lncRNAs were associated with the variation of fatty acid contents. Moreover, seven IncRNAs showed expression patterns positively correlated expression pattern with those of genes in de novo further research into lncRNAs that may regulate mesocarp development and lipid metabolism.

\section{Keywords}




\section{Introduction}

Noncoding RNAs (ncRNAs) constitute a critical part of the eukaryotic transcriptome and play a vital role in

gene regulation. Long noncoding RNAs (lncRNAs), an important class of noncoding RNAs, are non-protein coding

RNAs longer than $200 \mathrm{bp}$ and function as key regulators of diverse mechanisms in a range of biological processes

(Geisler \& Coller 2013; Rinn \& Chang 2012; Wang et al. 2011). Researchers have focused on the identification and characterization of lncRNAs responsible for biological process regulation over the past several decades. LncRNAs were classified into several groups based on their genomic location, including long intergenic noncoding RNAs (lincRNAs), intronic lncRNAs, natural antisense lncRNAs (NAT-lncRNAs) and sense lncRNAs (Kung et al. 2013; Rinn \& Chang 2012). Researchers have obtained numerous lncRNAs in plants, including NAT-lncRNAs in Arabidopsis (Zhao et al. 2018), lincRNAs in black cottonwood (Shuai et al. 2014), soybean and wheat (Golicz et al. 2017; Zhang et al. 2014), and lncRNAs associated with stress responses in cotton, grapevine, and Chinese cabbage (Jain et al. 2017; Wang et al. 2019a; Wang et al. 2019b; Wang et al. 2015; Xu et al. 2017; Yao et al. 2019). Oil palm (Elaeis guineensis) is an important oil crop in tropical and subtropical areas and its complete genome was released in 2013 (Singh et al. 2013). However, no reports on genome-wide lncRNA identification in oil palm are available. LncRNA loci serve as important regulatory mediators in gene expression and regulate the expression of target genes with cis-acting or trans-acting mechanisms (Kung et al. 2013; Liu et al. 2015b). LncRNAs can interact with multiple protein partners, serve as molecular scaffolds that help assemble and target the chromatin-modifying complex, and interact with miRNA as target mimics (Chu et al. 2011; Liu et al. 2012; Rinn \& Chang 2012; Vance \& Ponting 2014). For lncRNAs with cis-functions, their regulated genes are located close to the lncRNA loci (Herzog et al. 2014; Kim \& Shiekhattar 2016; Li et al. 2016). NAT-lncRNA loci may regulate their sense genes and influence their expression via diverse transcriptional or post-transcriptional mechanisms (Zhao et al. 2018). NATlncRNA loci may compete for RNA polymerase II and regulatory transcription factors with their sense genes and 
miRNA and complementary sequences between lncRNAs and target genes were used as criteria to select candidate target genes of lncRNAs (Jiang et al. 2015; Li et al. 2015; Wu et al. 2013; Zhao et al. 2018).

With the advance of next-generation sequencing (NGS) technology, RNA-seq has become the technical platform of choice to identify lncRNAs. Using bioinformatics tools, lncRNA transcripts can be directly assembled from RNA-seq reads. Recent advances in DNA sequencing and transcriptome analysis have provided gigabases of data and genome-wide analysis of IncRNAs has been conducted in many species (Jain et al. 2017; Ma et al. 2018; Wang et al. 2019a; Wang et al. 2019b; Xu et al. 2017; Yao et al. 2019). Approximately 40,000 putative lncRNAs were identified in Arabidopsis by EST, tiling array analyses and RNA-seq data sets (Jin et al. 2013; Liu et al. 2015a; Wang et al. 2014). Oil palm is one of the most important oil crops in the world. Many QTL mapping for oil yield, fatty acid composition and vegetative traits in oil palm were reported (Jeennor \& Volkaert 2014; Montoya et al. 2013; Singh et al. 2009), A good reference of oil palm genome, transcriptome profile and lncRNA profile will assist in identifying the causality for phenotypic variations. In this study, we applied strand-specific RNA-seq (ssRNA-seq) technology to identify lncRNA transcripts from an equally pooled RNA sample of oil palm spear leaf and mesocarps of six developmental stages. We also predicted target genes of lncRNAs and analysed the expression patterns of lncRNAs and their target genes based on 18 oil palm transcriptomes downloaded from the National Center for Biotechnology Information (NCBI) website. LncRNAs expression patterns in different tissues and different developmental stages of mesocarp that stores oil were characterized. We also applied association mapping to identify lncRNA loci related to the variation in fatty acid content. Our study provides a resource for studying lncRNAs in oil palm, including NAT-lncRNAs and their target genes, as well as lncRNAs related to fatty acid content.

9 Materials and Methods

\section{Plant materials}

One African oil palm plant (hybrid of dura and pisifera) sourced from Malaysia, and grown in the oil palm germplasm resources garden at Wenchang, Hainan, China were used for all experiments. The mesocarp for oil palm fruit at six developmental stages (8-week-old, 12-week-old, 16-week-old, 18-week-old, 20-week-old and 24-week- 
104 old), kernel, female flower, and spear leaves were collected from a ten-year-old oil palm individual (accession: CRI-

105 005). The mesocarp at six developing stages and spear leaves were used for the following ssRNA-seq. Roots were

106 collected from one-year-old oil palm seedlings that derived from the ten-year-old oil palm seeds, which were easier

107 to sample and not lignified as the ten-year-old oil palm plant. Each sample collection was done with three biological

108 replicates. All samples collected were immediately frozen in liquid nitrogen and stored $-80{ }^{\circ} \mathrm{C}$ until needed for RNA

109 extraction. All samples (mesocarp, kernel, female flower, spear leaves, and root) were used for the following

110 quantitative reverse transcription PCR (RT-qPCR) and RT-PCR.

111 A set of 18 oil palm transcriptomes downloaded from NCBI were also used in this study for gene expression

112 pattern analysis (Table S1). All these transcriptomes were derived from Illumina platform (paired-end, Illumina

113 HiSeq 2000) and included leaf (DRR053156), male flower (DRR053157), leaf from oil palm seedlings

114 (ERR1735779), female inflorescences at 6 leaf stage (SRR5189966), female inflorescences at 15 leaf stage

115 (SRR5189969), root from 3-month-old juvenile tree (SRR7812013, SRR7812014), and mesocarp at different

116 developmental stages, which included ERR1413765 (100 days after pollination (DAP)), ERR1413766 (140 DAP),

117 ERR1413767 (120 DAP), ERR1413768 (160 DAP), ERR3385821 (five months after pollination), ERR3385822

118 (five months after pollination), ERR3385823 (four months after pollination), ERR3385824 (five months after

119 pollination), ERR3385825 (four months after pollination), ERR3385826 (five months after pollination),

120 ERR3385828 (five months after pollination).

\section{RNA extraction, RNA-seq libary construction and strand-specific RNA sequencing}

122 Total RNA for oil palm mesocarps (six developing stages described above, with three biological replicates for 123 each stage) and spear leaves used for ssRNA-seq were extracted by a modified CTAB method (Xiao et al. 2012).

124 RNA degradation and contamination was detected by 1.5\% agarose gel electrophoresis. Total RNA concentration 125 and purity was determined using a Nanodrop ND-2000 spectrophotometer (Nanodrop Technologies, USA). RNA 126 integrity was verified using RNA Nano 6000 Kit for Agilent Bioanalyzer 2100. RNA samples without degradation 127 and contamination were used for rRNA removal. The RNA samples were equally pooled and a total amount of 1.5 128 ug RNA was treated with Ribo-Zero rRNA Removal Kit (epicentre, USA), and then fragmented by fragmentation 129 buffer. First-strand complementary DNA was synthesized using random hexamers and reverse transcriptase. 
130 Second-strand cDNA was prepared using RNase H, DNA polymerase I and dNTPs. Remaining overhangs were

131 converted into blunt ends via exonuclease/polymerase activities and 3' ends of DNA fragments were treated with

132 adenylation. After that, NEBNext adaptors were ligated to the ends of the prepared double-stranded cDNA and the

133 library fragments were purified by AMPure XP beads. The fragment size ranged from 150-200bp were selected and

134 PCR amplified, and then sequenced with an Illumina Hiseq 2000 platform by Biomarker Technology Co., Ltd

135 (Beijing, China). The clean reads from the above lncRNA-seq data were deposited in the European Bioinformatics

136 Institute (EMBL-EBI) at the European Nucleotide Archive (accession number: ERR3412516,

137 https://www.ebi.ac.uk/ena/data/view/ERR3412516).

138

139 RNA transcript assembly and novel transcriptional unit identification

140 We used the FastQC software (http://www.bioinformatics.babraham.ac.uk/projects/fastqc/) to check the

141 sequence quality of raw reads. Base quality value (Q) was estimated by the following formula: $\mathrm{Q}=-10 \times \log _{10} \mathrm{P}(\mathrm{P}$

142 represents the error probability during Illumina sequencing). Raw reads were pretreated to remove adaptor

143 sequences and low quality sequences via the Trimmomatic software (Bolger et al. 2014). The parameters for

144 Trimmomatic were set as: 1) remove adapters (ILLUMINACLIP:TruSeq3-PE.fa:2:30:10); 2) remove leading low

145 quality (LEADING:3), 3) remove trailing low quality (TRAILING:3); 4) scan the read with a 4-base wide sliding

146 window, cutting when the average quality per base drops below 15 (SLIDINGWINDOW:4:15); 5) drop reads below

147 the 36 bases long (MINLEN:36). Clean reads were obtained by removing reads containing adapter, reads containing

148 poly-N and low quality reads from raw data. After raw reads filtering, Q30 value of clean reads was above 95.88\%

149 and all reliable reads were mapped to the oil palm reference genome (Version EG5,

150 https:/www.ncbi.nlm.nih.gov/genome/2669) using hisat2 (version 2.1.0) with default parameters (Kim et al. 2015)

151 and de novo assembled using the StringTie software (version 2.0) with default parameters (Pertea et al. 2015). The

152 protein-coding transcripts identified in this study were annotated based on the oil palm gene prediction in NCBI

153 (Gene models based on file downloaded from the

154 website,ftp://ftp.ncbi.nlm.nih.gov/genomes/all/GCF/000/442/705/GCF_000442705.1_EG5/GCF_000442705.1_EG5

Peer) reviewing PDF | (2019:07:39449:4:0:NEW 27 May 2020) 
_genomic.gff.gz). After removing all annotated protein-coding genes, transcripts that had more than 200 bp in length, more than one exon and FPKM $\geq 0.1$ were selected for further identification of lncRNAs.

\section{IncRNAs identification, classification and characterization}

159 We removed transcripts that were likely to be assembly artifacts according to class code annotated by the

160 gffcompare program and retained transcripts annotated by "u", "i", "o" and "x", which represent novel intergenic,

161 intronic, sense-overlapped and cis-antisense transcripts, respectively (Trapnell et al. 2012). The transcripts of

162 candidate lncRNAs must not contain open reading frame encoding more than 50 amino acids and must not encode

163 any transposable elements. Coding Potential Calculator (CPC) (Kong et al. 2007), Coding-Non-Coding Index

164 (CNCI) (Sun et al. 2013), Coding Potential Assessment Tool (CPAT) (Wang et al. 2013) analysis and PfamScan

165 (Pfam 32 database) were applied to analyze transcripts. The assembled transcripts that did not pass the protein-

166 coding-score test (score $<0$ ) of $\mathrm{CPC}$ and $\mathrm{CNCI}$ analysis were as noncoding sequences. CPAT analyzed the open

167 reading frame length/coverage, Fickett score, and hexamer usage bias of the transcripts and determined the

168 noncoding sequences with default parameter. Based on PfamScan analysis, we eliminated transcript with potential

169 protein-coding ability (E-value cutoff $\leq 0.001$ ). The predicted long noncoding transcripts shared from the four

170 analyses were considered as candidate oil palm lncRNAs. We filtered out transposable elements for the transcripts

171 through PfamScan analysis and comparing with Dfam database (Hubley et al. 2015) through BLAST analysis (E-

172 value cutoff $<1 \mathrm{e}-5)$.

173 The different types of lncRNAs include lincRNA, intronic lncRNA, anti-sense lncRNA, sense lncRNA were

174 selected using cuffcompare (Trapnell et al. 2012). The protein-coding genes were derived from protein-coding

175 transcripts in this study, as well as oil palm predicted gene models downloaded from NCBI (Version: EG5). We

176 used StringTie to calculate Fragments Per Million Fragments (FPKM) values of lncRNAs and other protein-coding

177 genes. For gene with different isoforms, total FPKM values of all isoforms were used to represent the gene's FPKM

178 value.

179

180 
181

182

183

184

186

187

188

189

190

191

192

193

194

\section{Prediction of IncRNA target genes}

LncRNAs participated in regulatory pathways through two ways - in cis and in trans. Target genes for lncRNAs acting in cis were predicted by protein-coding genes overlapped within $2 \mathrm{~kb}$ flanking sequences of IncRNAs or overlapped with lncRNAs. The genomic positions of lncRNAs and protein-coding genes were compared to identify cis-acting target genes for lncRNA. We used the LncTar software to predict target genes in trans of lncRNA loci based on mRNA sequence complementary and RNA duplex energy prediction (Li et al. 2014).

\section{LncRNA expression profiles in different oil palm tissues and co-expression analysis}

A set of 18 oil palm RNA libraries were downloaded from the NCBI website (https://www.ncbi.nlm.nih.gov/) and used to calculate the fragments per kilobase of transcript per million fragments mapped (FPKM) for lncRNAs and their corresponding target genes (Table S1). Bowtie2 (Langmead \& Salzberg 2012) were used to map the reads to oil palm genomes and FPKM values were calculated by cufflinks (Trapnell et al. 2012).

Based on the reference of Zhao et al. (2018), Pearson Correlation Coefficients (p.c.c.) were calculated between the expression levels of adjacent protein-coding genes and between the expression levels of lncRNAs and their closest protein-coding genes. LncRNA/protein-coding gene pairs with low abundance $\left(\mathrm{FPKM}_{\max }<1\right)$ were excluded from our analysis. LncRNA/protein-coding gene pairs with Pearson correlation coefficients greater than 0.468 were presented in the heat map $(\mathrm{n}=16, \mathrm{P} \leq 0.05)$. The 18 transcriptome datasets described above were used for analysis of p.c.c. between lncRNAs and genes that belong to the pathways of plastid fatty acid synthesis from pyruvate and triacylglycerol (TAG) synthesis from the reference Xiao et al. (2019).

\section{Validation of the expression of IncRNAs and their target genes by RT-PCR and RT-qPCR}

The total RNAs for oil palm root, stem, spear leaf, female flower, kernel, and mesocarp tissues at six developing stages (8-week-old, 12-week-old, 16-week-old, 18-week-old, 20-week-old and 24-week-old) were extracted by a modified CTAB method as the above. The complementary DNA for each sample were synthesized using All-in-One First-Strand Synthesis MasterMix kit (NOVA, Jiangshu, China) with random hexamers, which was 
206

207

208

209

210

211

212

213

214

215

216

217

218

219

220

221

222

223

224

225

226

227

228

229

230

231

used for RT-PCR and RT-qPCR assays to validate the expression of lncRNAs and their target genes. We used Primer 5.0 to design the primers for these genes and listed the primer information in Table S2.

The RT-qPCR mixture contained $1 \mu$ diluted cDNA, $5 \mu$ of $2 \times$ FastStart Universal SYBR Green Master (NOVA, Jiangshu, China), and $0.5 \mu \mathrm{l}$ of each gene-specific primer $(10 \mu \mathrm{M})$ in a final volume of $10 \mu \mathrm{l}$. All PCR reactions were performed using ABI 7900HT machine under following conditions: 2 min at $95{ }^{\circ} \mathrm{C}$, and 40 cycles of $5 \mathrm{~s}$ at $95^{\circ} \mathrm{C}$ and $30 \mathrm{~s}$ at $60^{\circ} \mathrm{C}$ in $384-w e l l$ clear optical reaction plates (Applied Biosystems, USA). The procedure ended by a melt-curve ramping from 60 to $95{ }^{\circ} \mathrm{C}$ for 20 minutes to check the PCR specificity. All RT-qPCR reactions were carried out in biological and technical triplicate. A non-template control was also included in each run for each gene. The final $\mathrm{Ct}$ values were the means of nine values (biological triplicate, each in technical triplicate).

\section{Single nucleotide polymorphisms (SNPs) detection for IncRNAs and identification of IncRNAs related to} variation in fatty acid composition by association mapping

Based on 1,261,501 reliable SNPs markers (minor allele frequency $>0.05$ and more than $80 \%$ oil palm individuals had sequences information for each SNP marker) derived from SLAF sequencing in a diversity panel of 200 oil palm individuals in our previous research (Xia et al. 2019b), SNPs within lncRNA regions were screened by a Perl script. The contents of lauric acid (12:0), myristic acid (C14:0), palmitic acid (16:0), tripalmitelaidin acid (16:1), Hexadecadienoic acid (16:2), stearic acid (18:0), oleic acid (18:1), linoleic aicd (18:2), and oil for 160 individual out of 200 oil palm used in this study were the same set of data from our previous research (Xia et al. 2019a). Fatty acid composition was examined and measured using gas chromatography (Agilent DB-23, 30 m×250).

The nine values (three biological replicates $\times$ three technical replicates) obtained per oil palm individual were averaged for subsequent association mapping. The average values along with standard deviations of different fatty acids and oil contents for the 160 oil palm individuals were listed in Table S3. Since five subgroups for the 200 oil palm individuals were estimated based on cross-validation errors, mixed linear models (MLM) were used. Fixed effects were computed using a Q (population) value matrix, and random effects were computed using a K (kinship) matrix. The $\mathrm{Q}+\mathrm{K}$ value matrix was added to the MLM model. The $\mathrm{Q}$ matrix was obtained using STRUCTURE software (version 2.3.4) (Pritchard et al. 2000), and the K matrix (genetic relationships among the 200 oil palm 
232 individuals) was obtained using SPAGeDi software (version 1.5) (Hardy \& Vekemans 2002). Association analysis

233 was performed using Tassel 5.0 (Bradbury et al. 2007). P-values for associations between SNP markers and fatty

234 acid content were computed according to Yu et al (2006).

235

236

Results

237

Identification and characterization of oil palm IncRNAs

238 A total of 166,288,038 raw reads were generated from an equally pooled RNA sample of oil palm spear leaf, 8-

239 week-old, 12-week-old, 16-week-old, 18-week-old, 20-week-old and 24-week-old mesocarp tissues, and

$240165,257,052$ clean reads (24.69 Gbp) were obtained after adaptor trimming and sequence quality filtering.

241 Approximately $86.98 \%$ clean reads were mapped to the oil palm reference genome, and 96,009 transcripts were

242 assembled. To identify lncRNAs, we filtered out the assembled transcripts shorter than 200 bp and transcripts with

243 protein-coding potential via protein-coding-score test (CPC, CNCI, CPAT) and Pfam protein domain analysis,

244 which identified transcripts with potential protein-coding ability (cutoff E-value $\leq 0.001$ ). Finally, 1,663 transcripts

245 were tested as having no protein-coding potential and were considered candidate lncRNAs. These transcripts were

246 mapped to 1363 lncRNA loci and 289 lncRNA loci had more than one transcript (Table S4).

247 The 1,363 lncRNA loci were distributed across all 16 chromosomes (930) and unlinked scaffolds (433) of the

248 oil palm genome (Table 1). Chr1 (129), Chr2 (96) and Chr3 (95) had the largest number of lncRNA loci, while

249 Chr10 (31), Chr13 (32) and Chr15 (30) had the least number of lncRNA loci. Based on the present annotation

250 version of the oil palm genome, the 1,363 lncRNA loci were classified into four classes. LncRNA loci located in

251 intergenic regions (lincRNAs, 703) are the top number of lncRNA classes, followed by lncRNA loci located on the

252 antisense of protein coding genes (NAT-lncRNAs, 581), lncRNA loci overlapping with pseudogene regions (sense-

253 lncRNAs, 47) and lncRNA loci belonging to genic intronic regions (intronic, 32). Based on the genomic locations

254 for NAT-lncRNA loci and their corresponding sense genes, we further classified them into six types (Figure 1).

255 NAT-lncRNA loci for type I, II, III, IV overlapped in their genic regions with genes on their opposite strands, while

256 type V and type VI had promoter regions and 3' UTR regions overlapped with sense strand genes, respectively.

257 Expression profiles for identified oil palm IncRNAs

Peer] reviewing PDF | (2019:07:39449:4:0:NEW 27 May 2020) 
Based on the strand-specific RNA-seq data in this study, the expression levels for lncRNAs and protein-coding genes were compared. A large proportion for lncRNAs $(68 \%)$ and mRNAs $(62 \%)$ showed low expression levels (FPKM value $<1$ ), while the proportions of IncRNAs $(29 \%)$ and mRNAs $(31 \%)$ that had medium expression levels (FPKM: $1 \sim 15$ ) were close (Figure 2). For genes with high expression levels (FPKM $>15$ ), mRNA (7\%) had higher gene ratio than lncRNAs (1.6\%). Only 23 lncRNA loci had high expression levels (FPKM >15, Table S4).

To investigate the expression pattern of the identified IncRNAs in different tissues, we estimated the expression levels of each IncRNA by calculating FPKM in different tissues using the RNA-seq data from 18 samples for six tissues, including mesocarp (11), female flower (1), female inflorescences (1), male flower (1), leaf (2), and root (2) (Table S1). We excluded intronic-lncRNAs and NAT-lncRNAs whose transcripts overlapped with

267 protein-coding genes and used FPKM values for 916 lncRNAs for analysis, which are listed in Table S5. Among these transcriptomes, 77.8\% (ERR3385824, mesocarp) - 89.6\% (ERR1413765, mesocarp) of lncRNAs had low expression levels (FPKM values $0 \sim 1$ ) (Figure 3), $340 \operatorname{lncRNAs}$ were expressed (FPKM values $\geq 1$ ), and only 89 lncRNA loci had high expression levels (FPKM values $\geq 15$ ) in at least one tissue (Table S5). Among 89 lncRNA loci, $40 \%$ (36 loci) had high expression only in one transcriptome followed by $16 \%$ (14 loci) in two transcriptomes. There were ten lncRNA loci with high expression levels in more than ten transcriptomes (Table S5 and Figure 4). The number of lncRNA loci with high expression levels ranged from 12 (ERR3385826, mesocarp) to 36 (SRR5189966, female inflorescences) (Table S5). Comparing 23 loci identified in ssRNA-seq in this study, ten highly expressed IncRNAs (MSTRG.7397, MSTRG.10373, MSTRG.26953, MSTRG.32440, MSTRG.48470, MSTRG.51478, MSTRG.52125, MSTRG.52244, MSTRG.53207, and MSTRG.53370) were shared. Seven of the ten lncRNA loci were highly expressed in more than five transcriptomes (Figure 4). tissues, and 22 lncRNAs produced positive results (Table S2a, Figure 5 and Figure S1). Based on the FPKM values from the strand-specific sequence in this study, 47 out of 71 lncRNAs had relatively low expression (FPKM values $281 \leq 10$ ), and RT-PCR showed that 9 out of 47 loci produced positive bands in the tested samples (Table S2a, Figure 5 282 and Figure S1). In addition, RT-PCR showed that 10 out of 24 of the tested lncRNAs (FPKM values $>10$ ) had 
284

285

286

287

288

289

290

291

292

293

294

295

296

297

298

299

300

301

302

303

304

305

306

307

308

309

14737), MSTRG.48560 (FPKM = 72.8) and MSTRG.52384 (FPKM = 1.2) were expressed in all five tissues, while MSTRG.952 $(\mathrm{FPKM}=2465.9)$, MSTRG.7370 $(\mathrm{FPKM}=0.83)$, MSTRG.10334 $(\mathrm{FPKM}=3.13)$, MSTRG.36586 $(\mathrm{FPKM}=1.04)$ and MSTRG.48470 $(\mathrm{FPKM}=49.23)$ were expressed only in the mesocarp. The remaining 13 lncRNAs were expressed in two to four tissues.

\section{Target gene identification and coexpression analysis}

A total of 875 unique target genes were identified, including natural antisense lncRNAs (NAT-lncRNAs, 712), long intergenic noncoding RNAs (lincRNAs, 92), intronic-lncRNAs (33), and sense-lncRNAs (52) (Table S6). The flanking genes within a 2-kb distance and/or antisense overlapping genes (865) were identified as candidate targets in cis for lncRNAs, while 11 predicted target genes were predicted to be in trans. One gene LOC105051313, was also identified as a target gene, both in cis (MSTRG.23935) and in trans (MSTRG.23116). For NAT-lncRNAs, a total of 712 target genes were found, including target genes overlapping with NAT-lncRNAs on the opposite strand (657), located on the same strand of NAT-lncRNA within the 2-kb region (48), and mRNA sequences complementary to NAT-lncRNA via LncTar analysis (7) (Table S6). Of these target genes, 72\% (514) of target genes had genic regions overlapping with NAT-IncRNAs (class I, II, III, IV), and 144 target genes were located within the 2-kb flanking distance of NAT-lncRNAs, including 44 genes overlapping upstream promoter regions with NAT-lncRNAs (class V) and 100 genes overlapping for on the 3' downstream (class VI). For target genes overlapping with NAT-IncRNA (class I, II, III, IV), comparison of transcripts between NAT-lncRNAs and their target genes indicated that 263 pairs were overlapped and 287 pairs did not overlap (Table S6). The lincRNAs (703) were the top number type of identified lncRNAs, and we identified 87 target genes for 82 lincRNAs located within 2-kb regions (Table S6). Among these target genes, eight genes belong to lipid metabolism pathways by comparison with the genes in the pathways identified in our previous research (Table S6) (Xiao et al. 2019).

To explore the function of lncRNAs in the regulation of their target genes, we calculated the Pearson correlation coefficients (p.c.c.) between lncRNAs and their target genes. We identified 585 pairs of lncRNA loci and target genes that did not overlap in their transcripts (Table S7). Among these lncRNA/target pairs, 505 lncRNAs had one target gene and 66 lncRNAs had more than one target gene. For these lncRNAs in Table S7, a majority of 312

Peer] reviewing PDF | (2019:07:39449:4:0:NEW 27 May 2020) 
$310 \operatorname{lncRNAs}(250)$ had low expression $\left(0<\mathrm{FPKM}_{\max }<1\right)$ and $84 \operatorname{lncRNAs}$ had no detectable expression $\left(\mathrm{FPKM}_{\max }=0\right)$

311 in the 18 transcriptomes, while 47 target genes had low expression and 16 target genes with no detectable expression.

312 Since the existence of low expression levels or no expression among the 585 lncRNAs/target pair, 380 pairs of

313 lncRNA loci and target genes were filtered out. The p.c.c. for the remaining 205 pairs of lncRNAs/target genes were

314 examined and $10 \%$ (21) of the gene pairs showed positively correlated expression patterns (p.c.c. score $\geq 0.468$, $P$

315 value $<0.05$ ) are shown in Table S7. Only one gene pair - MSTRG.10328/LOC105041936 had negative correlation

316 relationship (p.c.c. score $=-0.61$, $\mathrm{P}$ value $<0.05$ ). For the 22 gene pairs, four lncRNAs (MSTRG.12377,

317 MSTRG.18648, MSTRG.47162, and MSTRG.26385) and one target gene (LOC105043335) had one transcriptome

318 with $\mathrm{FPKM}_{\max } \geq 1$, while the other gene in the pairs had $\mathrm{FPKM}_{\max } \geq 1$ in more than one transcriptomes (Table S7).

319 After RT-PCR analysis for expressed lncRNAs and target genes in the oil palm mesocarp, four NAT-lncRNAs and

320 five target genes proceeded for further RT-qPCR analysis. Positively correlated expression pattern for MSTRG.9572

321 / LOC105041276, MSTRG.13617 / LOC105044074 / LOC105044117, MSTRG.24786 / LOC105051990,

322 MSTRG.48560 / LOC109505170 (p.c.c. score > 0.9) were detected based on the RT-qPCR results (Figure 6).

323 Genetic variation in IncRNA loci and association with the variation of fatty acid composition

324 In a previous study, we developed 1,261,501 SNP markers for 200 oil palm individuals (Xia et al. 2019b) and determined the contents of lauric acid (12:0), myristic acid (C14:0), palmitic acid (16:0), tripalmitelaidin acid (16:1), Hexadecadienoic acid (16:2), stearic acid (18:0), oleic acid (18:1), linoleic acid (18:2), and oil for 160 out of 200 individuals (Xia et al. 2019a). Based on this set of data, 6,882 SNP markers were identified for the lncRNA regions.

328 A mixed linear model was used to analyse the association relationship between these SNP markers and the variation 329 in fatty acid compositions, and 28 SNP markers were significantly associated with the trait variation (cut-off of $\left.1 \mathrm{e}^{-3}\right)$.

330 One SNP marker (MSTRG.10380) had p value lower than 5.01e-08 $(-\log 10 \mathrm{p}=7.3)$ when based on $\mathrm{p}$ value

331 threshold in Xia et al. (2019a). These SNPs were located in 21 lncRNAs and associated with oil content (13) and

332 relative contents of palmitic aicd (6), linoliec acid (6), and stearic acid (3). The observed heterozygosity of the 28

333 SNP markers among 200 oil palm individuals varied from 0.1147 to 0.4968 with an average of 0.3414 (Table 2). A

334 majority of these SNPs (25) occured in the intron regions, while SNPs in MSTRG.2168, MSTRG.7396 and

335 MSTRG.15295 occured in the exon regions.

Peer) reviewing PDF | (2019:07:39449:4:0:NEW 27 May 2020) 
337 fatty acid synthesis and triacylglycerol (TAG) biosynthesis, 18 transcriptomes from mesocarp (11), female flower 338 (1), female inflorescences (1), male flower (1), leaf (2), and root (2) were used for calculating p.c.c. between these 339 genes. Seven lncRNAs had positively correlated expression patterns with 35 genes belonging to de novo synthesis of 340 fatty acids (27) and TAG synthesis (8) (Table S8). For the seven lncRNAs, MSTRG.15295 and MSTRG.30615 had $341 \mathrm{FPKM}_{\max } \geq 1$ in ten and eleven transcriptomes, respectively, while MSTRG.2123 and MSTRG.13525 had FPKM $\max$

$342 \geq 1$ in two transcriptomes and the other three lncRNAs had $\mathrm{FPKM}_{\max } \geq 1$ in one transcriptome (Table S8). Moreover, 343 a majory of correlated lipid-related genes (29) had FPKM $\mathrm{max}_{2} \geq 1$ in more than nine transcriptomes. Twenty-seven 344 genes in the de novo fatty acid synthesis pathway, including $E g P D H$ (5 genes), $E g A C C$ (7), $E g A C P 4$ (3), $E g K A R$ (1), $345 \operatorname{EgKASI}(2), \operatorname{EgKASIII}(1), \operatorname{EgER}$ (1), EgFatA (1), EgFatB1 (1), EgFatB3 (1), EgSAD-2 (1), EgHAD (2), and 346 EgWRI1-1 (1), were found to be positively correlated with seven lncRNAs. Among these genes, MSTRG.15295 347 positively correlated with the top number genes (17) with 15 genes in the de novo synthesis fatty acid pathway 348 followed by MSTRG.2123 (11), MSTRG.17117 (9), and MSTRG.16345 (5). The remaining three lncRNAs were 349 positively correlated with one to three genes positively. MSTRG.15295 had a positive correlation with stearoyl-ACP desaturase $(E g S A D)$ and a transcription factor - WRINKLED 1 (WRI 1).

351

\section{Discussion}

LncRNAs play important roles in mediating biological process regulation. In this study, we applied ssRNA-seq to identify lncRNAs in oil palm from mixed tissues of leaves and six different developmental stages of mesocarp.

355 Based on ssRNA-seq data in this study and 18 transcriptomes from other studies, we found that the majority 356 IncRNA loci had low expression. Coexpression analysis between lncRNAs and predicted target genes in cis 357 indicated that 21 lncRNA loci had positive correlations with target genes, while only one lncRNA locus displayed a 358 negative correlation. Based on the data of SNPs and fatty acid content data from our previous research, 28 SNPs 359 belonging to 21 lncRNAs were associated with fatty acid composition. Our study identified a collection of lncRNAs 360 for oil palm and provided clues for further investigations into lncRNAs that may regulate mesocarp development 361 and lipid metabolism. 
362 Data mining for lncRNAs was feasible since a large amount of transcription data was available. In model

363 plants, such as Arabidopsis, maize and rice, a large quantity of IncRNA loci were identified (Li et al. 2014; Zhang et

364 al. 2014; Zhao et al. 2018), some lncRNAs were validated and play critical roles in flowering controls, grain yield,

365 stress response and other biological processes (Jain et al. 2017; Jiang et al. 2019; Kindgren et al. 2018; Ma et al.

366 2018; Wang et al. 2018). LincRNAs are the most abundant lncRNA types (Table 1), while the majority of lncRNAs

367 had a relatively lower expression than did protein-coding genes (Figure 2 and Figure 3). These are common

368 phenomena for lncRNAs in many species (Wang et al. 2015; Yao et al. 2019). We used mixed samples to conduct

369 ssRNA-seq, which could cover more lncRNA transcripts. The downloaded 18 transcriptomes showed similar low

370 expression levels for most lncRNAs, while lncRNAs highly expressed in more samples tended to be detected in

371 different transcriptomes. The RT-PCR results fit the transcriptome data, 22 out 71 selected lncRNAs were expressed

372 in the tested tissues and four lncRNAs were expressed specifically in the mesocarp.

373 LncRNAs regulate target genes by serving as target mimics of miRNAs and regulators of transcripion and

374 chromatin modification (De Lucia \& Dean 2011; Jiang et al. 2019; Magistri et al. 2012; Wang et al. 2018; Wu et al.

375 2013; Zhao et al. 2018). Research in Arabidopsis on NAT-IncRNAs demonstrated that NAT-lncRNAs often

376 positively correlate with their cognate sense genes (Zhao et al. 2018). Coexpression analysis in this study also

377 suggested that lncRNAs may cotranscribed with adjacent target genes and $6 \%$ of $\operatorname{lncRNA} / \operatorname{target}$ pairs showed a

378 positive correlation in expression (Table S7). The positive correlation results for four lncRNAs and their target

379 genes were also validated by RT-qPCR (Figure 6). However, since a majority of lncRNA loci had low expression

380 levels, our results suggested that a small proportion of target genes may be cotranscribed with lncRNAs in cis.

381 Oil palm has $90 \%$ oil in its mesocarp, the highest level observed in the plant kingdom. Bourgis et al (2011)

382 used RNA-seq to examine transcriptional changes during oil palm mesocarp development, and found that synthesis

383 of fatty acids and supply of pyruvate in the plastid were the major factors controlling oil storage in the oil palm

384 mesocarp (Bourgis et al. 2011). Researchers have used QTL mapping and association mapping to identify key loci

385 related to the content or composition of fatty acids in oil palm composition (Jeennor \& Volkaert 2014; Montoya et al.

386 2013; Singh et al. 2009). In our study, we used association mapping and found 21 lncRNAs related to the variation

387 of fatty acid composition and oil content of oil palm mesocarp (Table 2). The coexpression analysis between the 21

Peer] reviewing PDF | (2019:07:39449:4:0:NEW 27 May 2020) 
388 lncRNAs and genes belonging to fatty acid synthesis and TAG synthesis pathways showed that nine lncRNAs had a

389 similar expression patterns as genes in the two lipid metabolism pathways. MSTRG.15295 had similar expression

390 pattern with the majority of correlated genes, including a key transcription factor - WRII. The WRI1 gene is

391 considered to play an important role in oil accumulation (Bourgis et al. 2011; Cernac \& Benning 2004; Dussert et al.

392 2013; Focks \& Benning 1998; Troncoso-Ponce et al. 2011). Our results provide candidate lncRNA loci related to the

393 development and oil storage of oil palm mesocarp.

\section{Conclusions}

395 This study provided an important collection of lncRNAs in oil palm and a set of 581 NAT-lncRNAs and 712

396 targets were predicted. Based on 18 oil palm transciptomes, we found that A $77.8 \%-89.6 \%$ lncRNA loci had low

397 expression, while only 89 lncRNA loci had medium to high expression in at least one transcriptome. Coexpression

398 analysis between lncRNAs and their target genes indicated that $10 \%$ of lncRNAs had positively correlated

399 expression patterns with target genes. Based on SNP markers derived from our previous research, 28 SNPs

400 belonging to $21 \operatorname{lncRNAs}$ were associated with the variation of fatty acid contents. Moreover, twelve lncRNAs

401 showed positively correlated expression patterns with genes in de novo fatty acid synthesis pathways. Our study

402 identified a collection of $\operatorname{lncRNAs}$ for oil palm and provided clues for further research investigating lncRNAs that

403 may regulate mesocarp development and lipid metabolism.

404

405

Reference

406 Bolger A, Lohse M, and Usadel B. 2014. Trimmomatic: a flexible trimmer for Illumina sequence data.

407 Bioinformatics 30:2114-2120.

408 Bourgis F, Kilaru A, Cao X, Ngando-Ebongue G-F, Drira N, Ohlrogge JB, and Arondel V. 2011. Comparative 409 transcriptome and metabolite analysis of oil palm and date palm mesocarp that differ dramatically in carbon $410 \quad$ partitioning. Proc Natl Acad Sci USA 108:12527-12532. 10.1073/pnas.1106502108

411 Bradbury PJ, Zhang Z, Kroon D, Casstevens TM, Ramdoss Y, and Buckler ES. 2007. TASSEL: software for 412 association mapping of complex traits in diverse samples. Bioinformatics 23:2633-2635. 
413 Cernac A, and Benning C. 2004. WRINKLED1 encodes an AP2/EREB domain protein involved in the control of $414 \quad$ storage compound biosynthesis in Arabidopsis. Plant J 40:575-585. TPJ2235 [pii]

$415 \quad 10.1111 / \mathrm{j} .1365-313 X .2004 .02235 . \mathrm{x}$

416 Chu C, Qu K, Zhong F, Artandi SE, and Chang HY. 2011. Genomic maps of long noncoding RNA occupancy reveal principles of RNA-chromatin interactions. Molecular cell 44:667-678.

De Lucia F, and Dean C. 2011. Long non-coding RNAs and chromatin regulation. Current Opinion in Plant Biology $14: 168-173$.

420

421

422

423

424

425

426

427

428

429

430

431

432

433

434

435

436

437

Dussert S, Guerin C, Andersson M, Joët T, Tranbarger TJ, Pizot M, Sarah G, Omore A, Durand-Gasselin T, and Morcillo F. 2013. Comparative transcriptome analysis of three oil palm fruit and seed tissues that differ in oil content and fatty acid composition. Plant Physiol 162:1337-1358.

Faghihi MA, and Wahlestedt CR. 2009. Regulatory roles of natural antisense transcripts. Nature Reviews Molecular Cell Biology 10:637-643.

Focks N, and Benning C. 1998. wrinkled1: A novel, low-seed-oil mutant of Arabidopsis with a deficiency in the seed-specific regulation of carbohydrate metabolism. Plant Physiol 118:91-101.

Geisler S, and Coller J. 2013. RNA in unexpected places: long non-coding RNA functions in diverse cellular contexts. Nature Reviews Molecular Cell Biology 14:699-712.

Golicz AA, Singh M, and Bhalla PL. 2017. The long intergenic non-coding RNA (lincRNA) landscape of the soybean genome. Plant Physiol 176:2133-2147.

Hardy OJ, and Vekemans X. 2002. spagedi: a versatile computer program to analyse spatial genetic structure at the individual or population levels. Molecular Ecology Notes 2:618-620. 10.1046/j.1471-8286.2002.00305.x

Herzog VA, Lempradl A, Trupke J, Okulski H, Altmutter C, Ruge F, Boidol B, Kubicek S, Schmauss G, and Aumayr K. 2014. A strand-specific switch in noncoding transcription switches the function of a Polycomb/Trithorax response element. Nature genetics 46:973-981.

Hubley R, Finn RD, Clements J, Eddy SR, Jones TA, Bao W, Smit AFA, and Wheeler TJ. 2015. The Dfam database of repetitive DNA families. Nucleic Acids Research 44:D81-D89. 10.1093/nar/gkv1272 
438

439

440

441

442

443

444

445

446

447

448

449

450

451

452

453

454

455

456

457

458

459

460

461

462

463

Jain P, Sharma V, Dubey H, Singh P, Kapoor R, Kumari M, Singh J, Pawar DV, Bisht DS, and Solanke AU. 2017. Identification of long non-coding RNA in rice lines resistant to rice blast pathogen Maganaporthe oryzae. Bioinformation 13:249-255.

Jeennor S, and Volkaert H. 2014. Mapping of quantitative trait loci (QTLs) for oil yield using SSRs and gene-based markers in African oil palm (Elaeis guineensis Jacq.). Tree Genetics \& Genomes 10:1-14.

Jiang N, Cui J, Shi Y, Yang G, Zhou X, Hou X, Meng J, and Luan Y. 2019. Tomato lncRNA23468 functions as a competing endogenous RNA to modulate $N B S-L R R$ genes by decoying miR482b in the tomatoPhytophthora infestans interaction. Horticulture Research 6:28. 10.1038/s41438-018-0096-0

Jiang Q, Ma R, Wang J, Wu X, Jin S, Peng J, Tan R, Zhang T, Li Y, and Wang Y. 2015. LncRNA2Function: a comprehensive resource for functional investigation of human lncRNAs based on RNA-seq data. $B M C$ Genomics 16:1-11.

Jin J, Liu J, Wang H, Wong L, and Chua N. 2013. PLncDB: plant long non-coding RNA database. Bioinformatics 29:1068-1071.

Kim D, Langmead B, and Salzberg SL. 2015. HISAT: a fast spliced aligner with low memory requirements. Nature Methods 12:357-360.

Kim TK, and Shiekhattar R. 2016. Diverse regulatory interactions of long noncoding RNAs. Current Opinion in Genetics \& Development 36:73-82.

Kindgren P, Ard R, Ivanov M, and Marquardt S. 2018. Transcriptional read-through of the long non-coding RNA SVALKA governs plant cold acclimation. Nature Communications 9:4561. 10.1038/s41467-018-07010-6

Kong L, Zhang Y, Ye Z-Q, Liu X-Q, Zhao S-Q, Wei L, and Gao G. 2007. CPC: assess the protein-coding potential of transcripts using sequence features and support vector machine. Nucleic Acids Research 35:W345-W349. $10.1093 / \mathrm{nar} / \mathrm{gkm} 391$

Kung JT, Colognori D, and Lee JT. 2013. Long noncoding RNAs: past, present, and future. Genetics 193:651-669.

Langmead B, and Salzberg SL. 2012. Fast gapped-read alignment with Bowtie 2. Nature Methods 9:357-359.

Li J, Ma W, Zeng P, Wang J, Geng B, Yang J, and Cui Q. 2015. LncTar: a tool for predicting the RNA targets of long noncoding RNAs. Briefings in Bioinformatics 16:806-812.

Peer] reviewing PDF | (2019:07:39449:4:0:NEW 27 May 2020) 
464 Li L, Eichten S, Shimizu R, Petsch K, Yeh C-T, Wu W, Chettoor A, Givan S, Cole R, Fowler J, Evans MM,

465

466

467

468

469

470

471

472

473

474

475

476

477

478

479

480

481

482

483

484

485

486

487

488

Scanlon M, Yu J, Schnable P, Timmermans MC, Springer N, and Muehlbauer G. 2014. Genome-wide discovery and characterization of maize long non-coding RNAs. Genome Biology 15:R40.

Li R, Zhu H, and Luo Y. 2016. Understanding the functions of long non-coding RNAs through their higher-order structures. International Journal of Molecular Sciences 17:702.

Liu J, Jung C, Xu J, Wang H, Deng S, Bernad L, Arenashuertero C, and Chua N. 2012. Genome-wide analysis uncovers regulation of long intergenic noncoding RNAs in Arabidopsis. The Plant Cell 24:4333-4345.

Liu J, Wang H, and Chua N. 2015a. Long noncoding RNA transcriptome of plants. Plant biotechnology journal 13:319-328.

Liu X, Hao L, Li D, Zhu L, and Hu S. 2015b. Long non-coding RNAs and their biological roles in plants. Genomics, Proteomics \& Bioinformatics 13:137-147.

Ma K, Shi W, Xu M, Liu J, and Zhang F. 2018. Genome-wide identification and characterization of long non-coding RNA in wheat roots in response to Ca2+ channel blocker. Frontiers in Plant Science 9.

Magistri M, Faghihi MA, Laurent GS, and Wahlestedt CR. 2012. Regulation of chromatin structure by long noncoding RNAs: focus on natural antisense transcripts. Trends in Genetics 28:389-396.

Montoya C, Lopes R, Flori A, Cros D, Cuellar T, Summo M, Espeout S, Rivallan R, Risterucci A, and Bittencourt D. 2013. Quantitative trait loci (QTLs) analysis of palm oil fatty acid composition in an interspecific pseudobackcross from Elaeis oleifera (H.B.K.) Cortés and oil palm (Elaeis guineensis Jacq.). Tree Genetics \& Genomes 9:1207-1225.

Pertea M, Pertea G, Antonescu C, Chang TC, Mendell JT, and Salzberg SL. 2015. StringTie enables improved reconstruction of a transcriptome from RNA-seq reads. Nature biotechnology 33:290-295.

Pritchard JK, Stephens M, and Donnelly P. 2000. Inference of population structure using multilocus genotype data. Genetics 155:945-959.

Rinn JL, and Chang HY. 2012. Genome regulation by long noncoding RNAs. Annual Review of Biochemistry $81: 145-166$.

Peer] reviewing PDF | (2019:07:39449:4:0:NEW 27 May 2020) 
489

490

491

492

493

494

495

496

497

498

499

500

501

502

503

504

505

506

507

508

509

510

511

512

513

514

Shuai P, Liang D, Tang S, Zhang Z, Ye C, Su Y, Xia X, and Yin W. 2014. Genome-wide identification and functional prediction of novel and drought-responsive lincRNAs in Populus trichocarpa. Journal of Experimental Botany 65:4975-4983.

Singh R, Ongabdullah M, Low ETL, Manaf MAA, Rosli R, Nookiah R, Ooi LCL, Ooi SE, Chan KL, and Halim MAA. 2013. Oil palm genome sequence reveals divergence of interfertile species in old and new worlds. Nature 500:335-339.

Singh R, Tan SG, Panandam JM, Rahman RA, Ooi LCL, Low EL, Sharma M, Jansen J, and Cheah S. 2009. Mapping quantitative trait loci (QTLs) for fatty acid composition in an interspecific cross of oil palm. BMC Plant Biology 9:114-114.

Sun L, Luo H, Bu D, Zhao G, Yu K, Zhang C, Liu Y, Chen R, and Zhao Y. 2013. Utilizing sequence intrinsic composition to classify protein-coding and long non-coding transcripts. Nucleic Acids Research 41.

Trapnell C, Roberts A, Goff L, Pertea G, Kim D, Kelley DR, Pimentel H, Salzberg SL, Rinn JL, and Pachter L. 2012. Differential gene and transcript expression analysis of RNA-seq experiments with TopHat and Cufflinks. Nature Protocols 7:562. 10.1038/nprot.2012.016

Troncoso-Ponce MA, Kilaru A, Cao X, Durrett TP, Fan J, Jensen JK, Thrower NA, Pauly M, Wilkerson C, and Ohlrogge JB. 2011. Comparative deep transcriptional profiling of four developing oilseeds. Plant $J$ 68:1014-1027. 10.1111/j.1365-313X.2011.04751.x

Vance KW, and Ponting CP. 2014. Transcriptional regulatory functions of nuclear long noncoding RNAs. Trends in Genetics 30:348-355.

Wang A, Hu J, Gao C, Chen G, Wang B, Lin C, Song L, Ding Y, and Zhou G. 2019a. Genome-wide analysis of long non-coding RNAs unveils the regulatory roles in the heat tolerance of Chinese cabbage (Brassica rapa ssp.chinensis). Scientific Reports 9:5002. 10.1038/s41598-019-41428-2

Wang H, Chung PJ, Liu J, Jang I, Kean MJ, Xu J, and Chua N. 2014. Genome-wide identification of long noncoding natural antisense transcripts and their responses to light in Arabidopsis. Genome Research 24:444-453.

Wang L, Park HJ, Dasari S, Wang S, Kocher J, and Li W. 2013. CPAT: coding-potential assessment tool using an alignment-free logistic regression model. Nucleic Acids Research 41. 
515 Wang P, Dai L, Ai J, Wang Y, and Ren F. 2019b. Identification and functional prediction of cold-related long noncoding RNA (lncRNA) in grapevine. Scientific Reports 9:6638. 10.1038/s41598-019-43269-5

517 Wang T, Liu M, Zhao M, Chen R, and Zhang W. 2015. Identification and characterization of long non-coding RNAs involved in osmotic and salt stress in Medicago truncatula using genome-wide high-throughput sequencing. BMC Plant Biology 15:131-131.

Wang X, Song X, Glass CK, and Rosenfeld MG. 2011. The long arm of long noncoding RNAs: roles as sensors regulating gene transcriptional programs. Cold Spring Harbor Perspectives in Biology 3.

Wang Y, Luo X, Sun F, Hu J, Zha X, Su W, and Yang J. 2018. Overexpressing lncRNA LAIR increases grain yield and regulates neighbouring gene cluster expression in rice. Nature Communications 9:3516. $10.1038 / \mathrm{s} 41467-018-05829-7$

Wight M, and Werner A. 2013. The functions of natural antisense transcripts. Essays in Biochemistry 54:91-101.

Wu H, Wang Z, Wang M, and Wang X. 2013. Widespread long noncoding RNAs as endogenous target mimics for microRNAs in plants. Plant Physiol 161:1875-1884.

528 Xia W, Luo T, Dou Y, Zhang W, Mason AS, Huang D, Huang X, Tang W, Wang J, Zhang C, and Xiao Y. 2019 a. Identification and validation of candidate genes involved in fatty acid content in oil palm by genome-wide association analysis. Frontiers in Plant Science 10. 10.3389/fpls.2019.01263

531 Xia W, Luo T, Zhang W, Mason AS, Huang D, Huang X, Tang W, Dou Y, Zhang C, and Xiao Y. 2019 b. Development of high-density SNP markers and their application in evaluating genetic diversity and population structure in Elaeis guineensis. Frontiers in Plant Science 10. 10.3389/fpls.2019.00130

Xiao Y, Xia W, Mason AS, Cao Z, Fan H, Zhang B, Zhang J, Ma Z, Peng M, and Huang D. 2019. Genetic control of fatty acid composition in coconut (Cocos nucifera), African oil palm (Elaeis guineensis), and date palm (Phoenix dactylifera). Planta 249:333-350. 10.1007/s00425-018-3003-x Plant Biology 17:42. 
540 Yao Z, Chen Q, Chen D, Zhan L, Zeng K, Gu A, Zhou J, Zhang Y, Zhu Y, Gao W, Wang L, Zhang Y, and Qu Y.

541 2019. The susceptibility of sea-island cotton recombinant inbred lines to Fusarium oxysporum $f$. sp.

542 vasinfectum infection is characterized by altered expression of long noncoding RNAs. Scientific Reports

$543 \quad 9: 2894.10 .1038 / \mathrm{s} 41598-019-39051-2$

544 Yu J, Pressoir G, Briggs WH, Bi IV, Yamasaki M, Doebley J, Mcmullen MD, Gaut BS, Nielsen DM, and Holland

545 JB. 2006. A unified mixed-model method for association mapping that accounts for multiple levels of

$546 \quad$ relatedness. Nature genetics 38:203-208.

547 Zhang Y, Liao J, Li Z, Yu Y, Zhang J, Li Q, Qu L, Shu W, and Chen Y. 2014. Genome-wide screening and

548 functional analysis identify a large number of long noncoding RNAs involved in the sexual reproduction of $549 \quad$ rice. Genome Biology 15:512-512.

550 Zhao X, Li J, Lian B, Gu H, Li Y, and Qi Y. 2018. Global identification of Arabidopsis lncRNAs reveals the 551 regulation of MAF4 by a natural antisense RNA. Nature Communications 9:5056. 10.1038/s41467-018-

552 $07500-7$ 
Figure 1

Figure 1 Classification of NAT-IncRNAs 
Overlapping NAT-lncRNA(n=468)

$\rightarrow$ Protein coding gene

$\rightarrow$ NAT-lncRNA

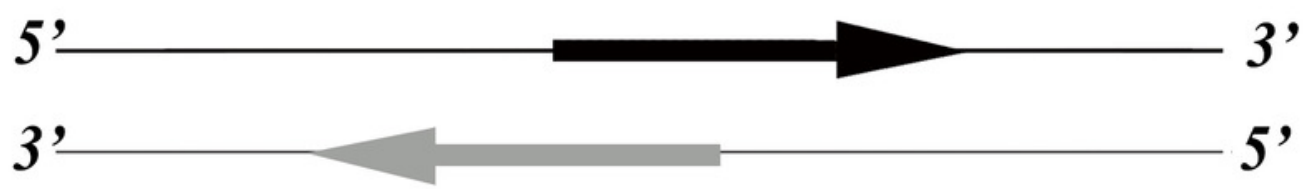

I

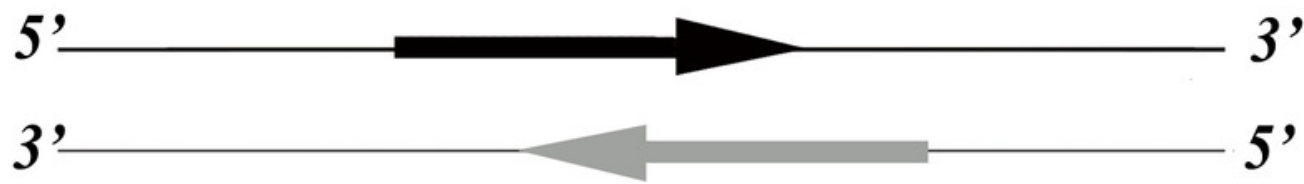

II

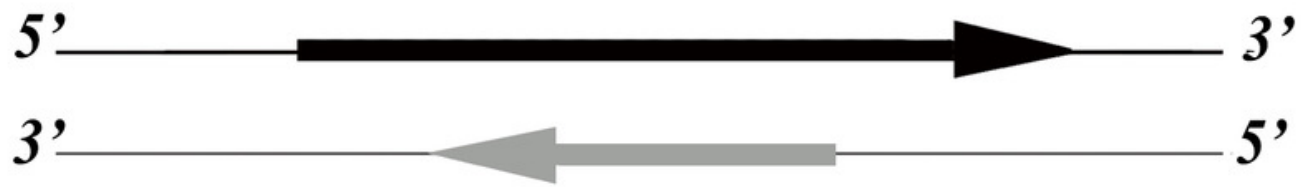

III

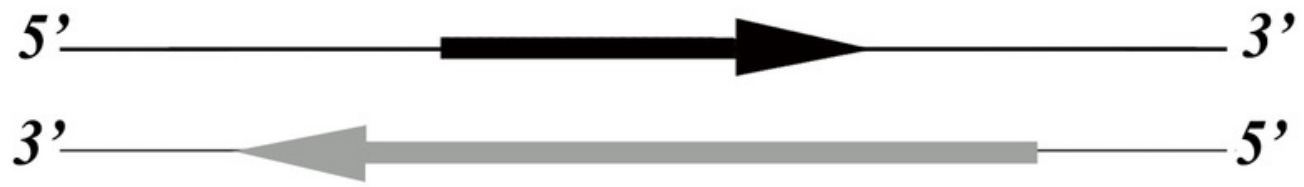

IV

Non-overlapping NAT- $\operatorname{lncRNA}(\mathrm{n}=113)$
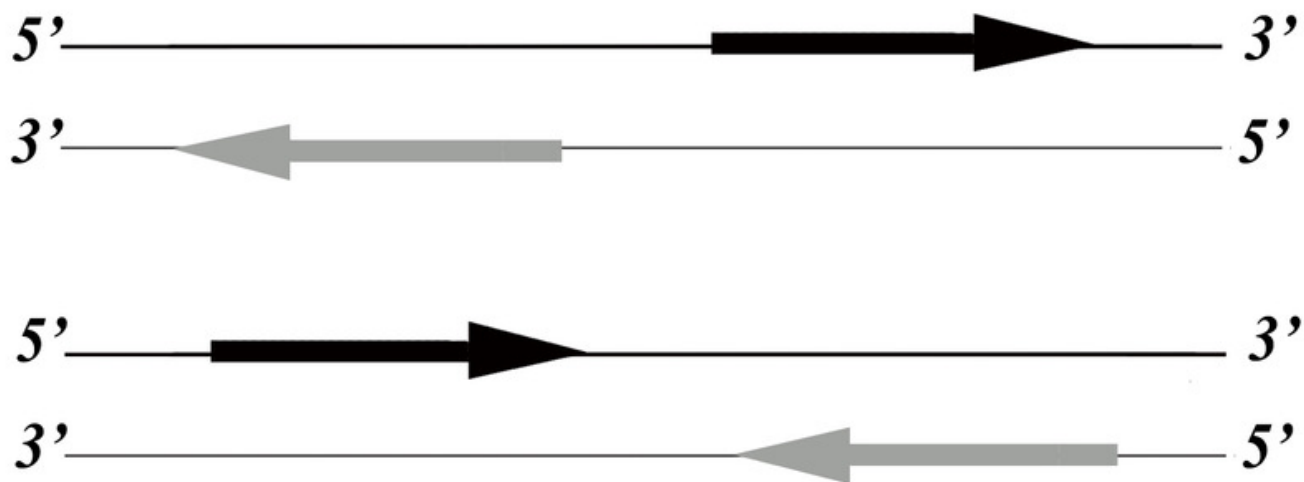

VI 
Figure 2

Figure 2 FPKM values comparison between IncRNA and mRNA.

A) Box-plots for $\log _{10}$ transformed FPKM values; B) IncRNA/mRNA percentages for low expression level (0-1), median expression level (1-15) and high expression levels genes $(15-100$ and $>100)$.
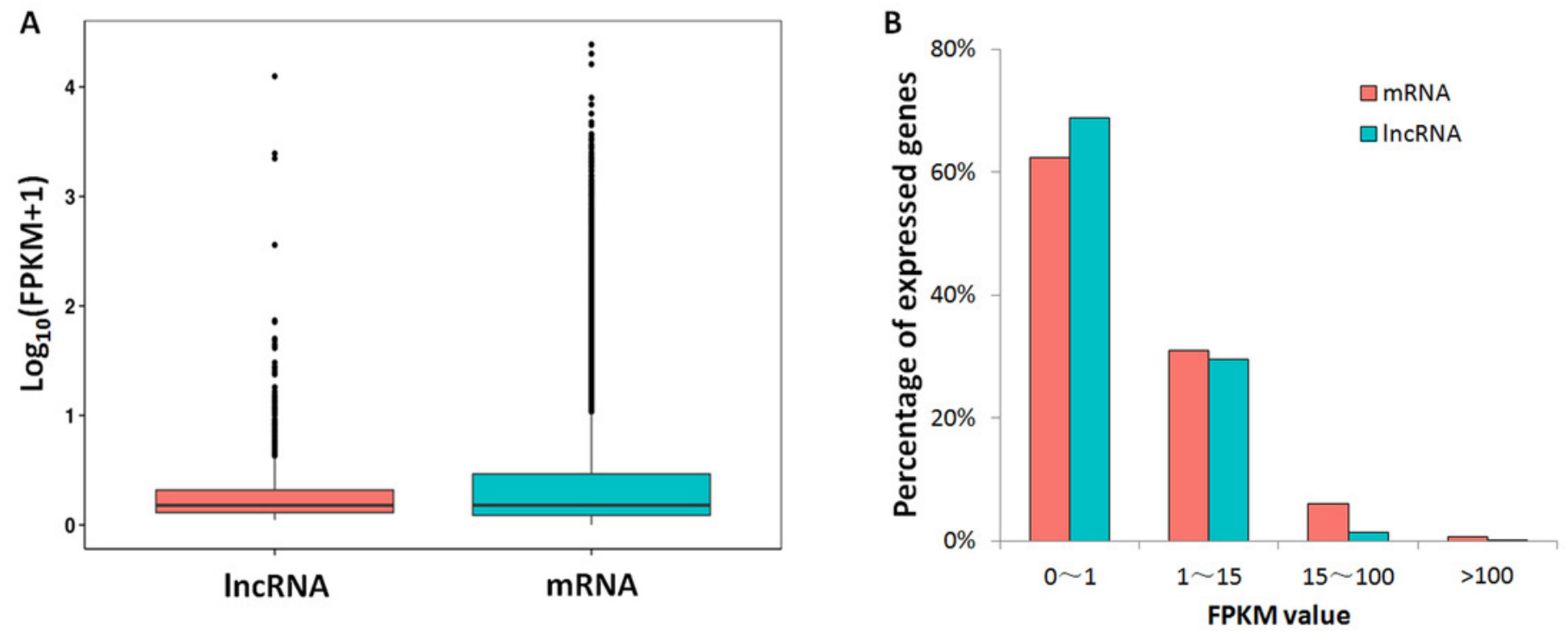


\section{Figure 3}

Figure 3 FPKM values distribution of IncRNA loci in 18 oil palm transcriptomes.

IncRNA percentages for low expression level (0-1), median expression level (1-15) and high expression levels genes (15-100 and >100).

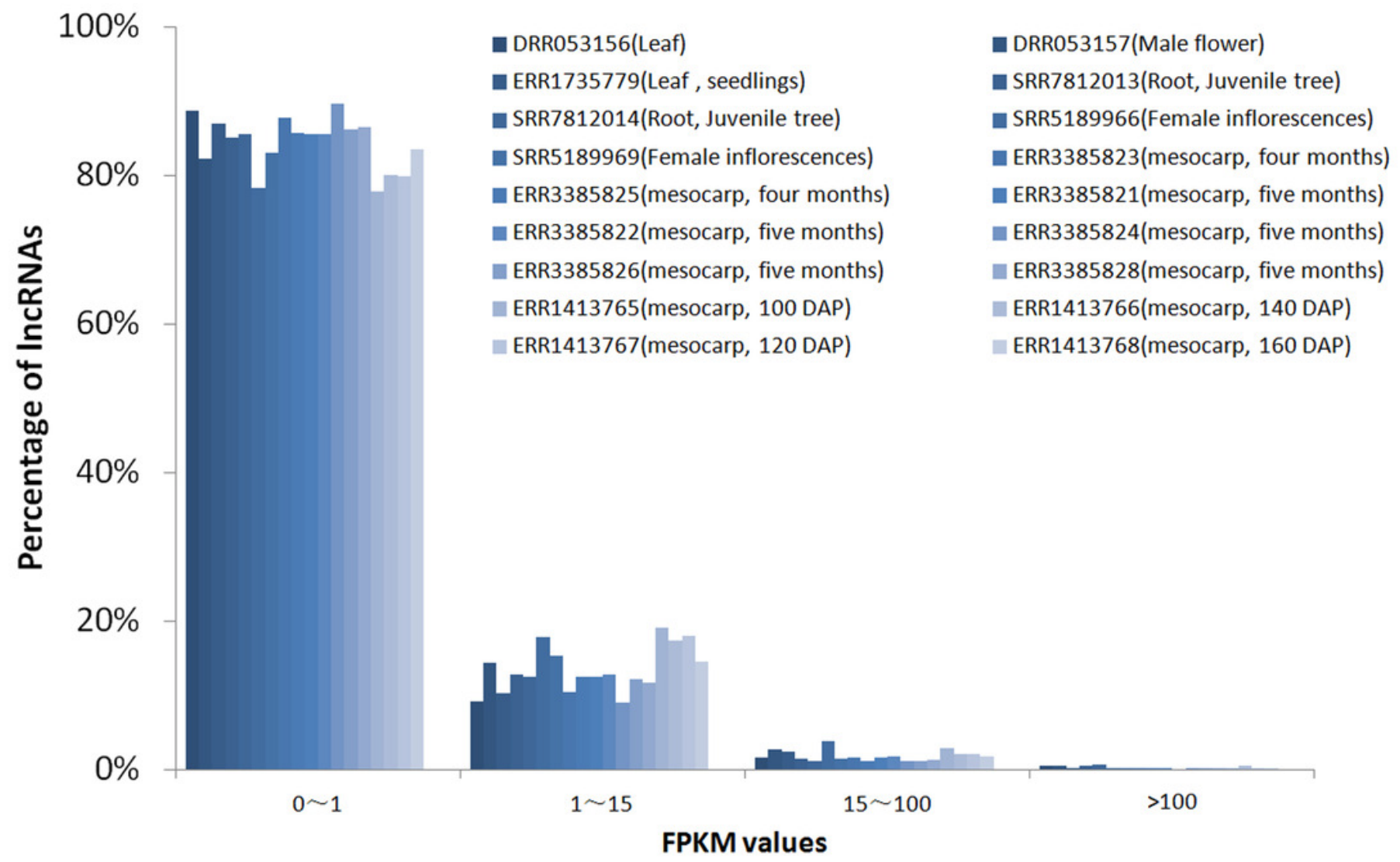




\section{Figure 4}

Figure 4 Heatmap for IncRNAs in 18 oil palm transcriptomes.

FPKM values for each IncRNA was used for K-means clustering and displayed according to the appearance number of high expression level (FPKM $\geq 15)$ in 18 transcriptome. LncRNA loci with arrows were highly expressed in both the ssRNA-seq result in this study and in the 18 transcriptomes. 


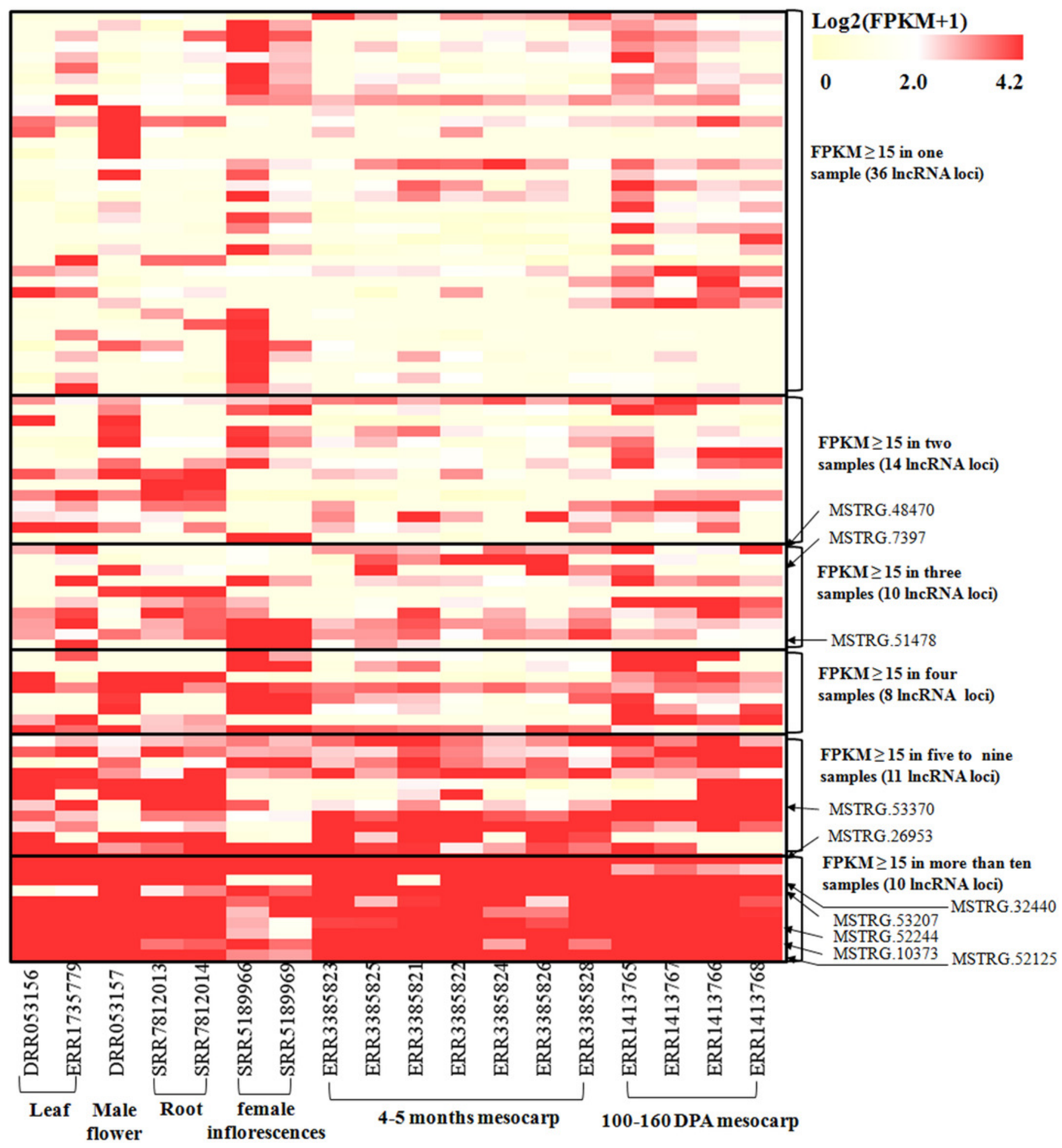


Figure 5

Figure 5 RT-PCR validation of 11 oil palm IncRNAs in six developing stages of mesocarp, leaf, root, flower and kernel of oil palm.

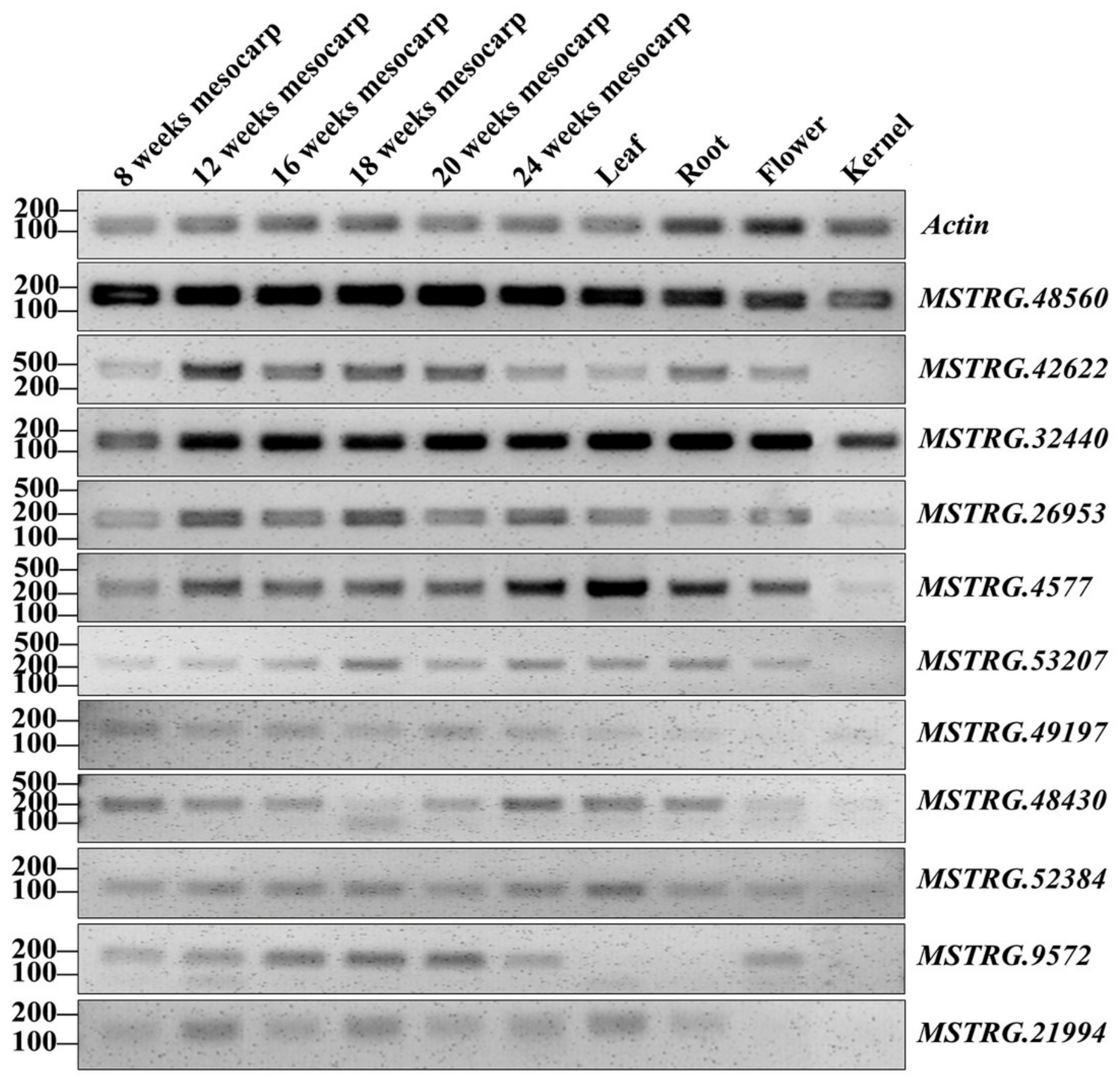


Figure 6

Figure 6 RT-qPCR validation of four oil palm IncRNAs and their target genes in six developing stages of mesocarp, leaf, root, flower and kernel of oil palm.

Pairs of oil palm IncRNA and their target genes included A) MSTRG.9572/LOC105041276; B) MSTRG.13617/ LOC105044117 and MSTRG.13617/ LOC105044074; C) MSTRG.48560/ LOC105051990; and D) MSTRG.24786/ LOC109505170.
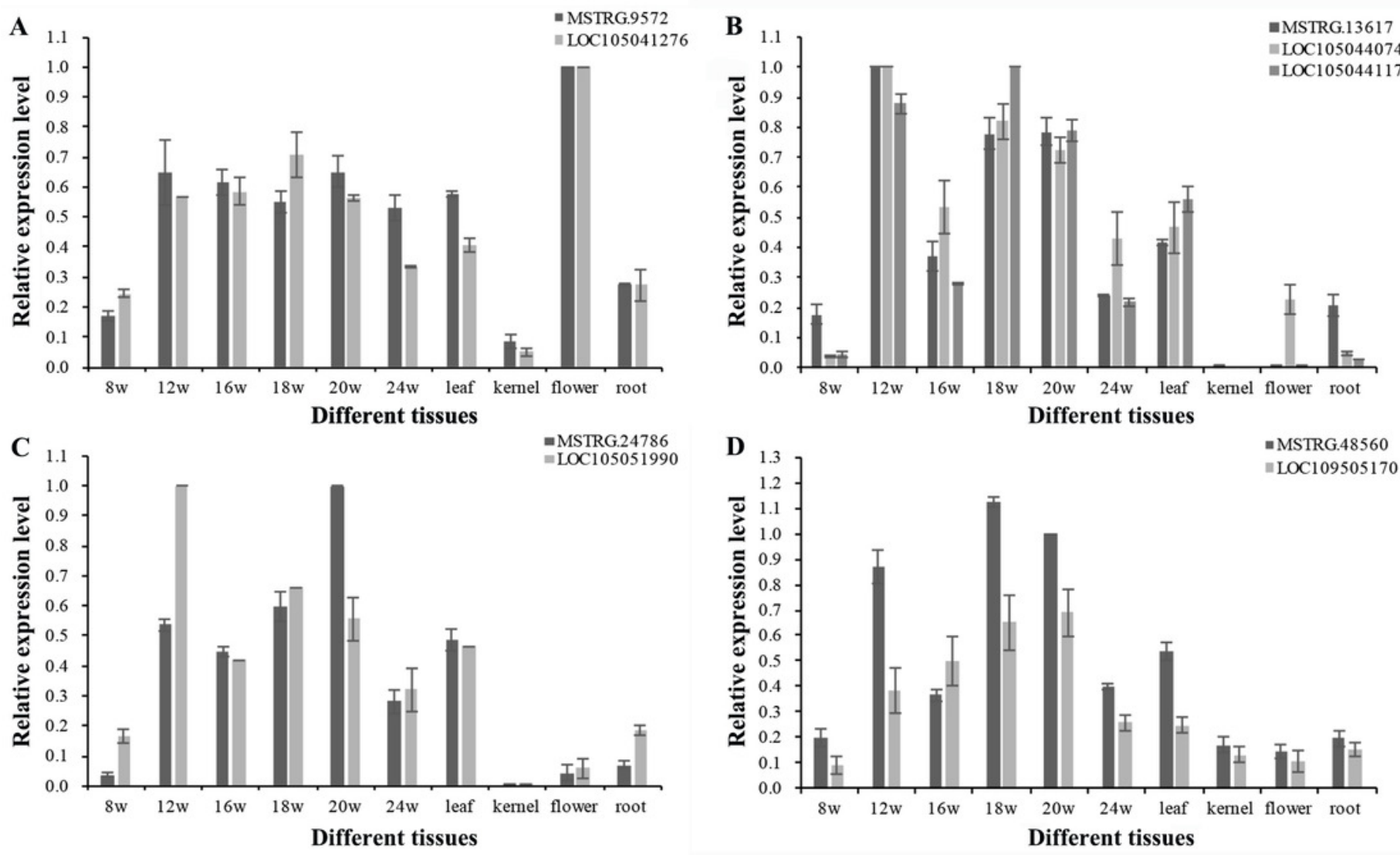


\section{Table $\mathbf{1}$ (on next page)}

Table 1 Genomic distribution of four IncRNA types across the African oil palm genome.

Genomic distribution of four IncRNA types across the African oil palm genome. 
1 Table 1 Genomic distribution of four lncRNA types across the African oil palm genome.

\begin{tabular}{lccccc}
\hline Chromosome & LincRNA & NAT-IncRNA & Intronic-lncRNA & Sense-lncRNA & total \\
\hline Chr1 & 61 & 59 & 4 & 5 & 129 \\
Chr2 & 44 & 47 & 2 & 3 & 96 \\
Chr3 & 46 & 46 & 1 & 2 & 95 \\
Chr4 & 38 & 41 & 1 & 4 & 84 \\
Chr5 & 26 & 45 & 1 & 3 & 75 \\
Chr6 & 24 & 31 & 1 & 2 & 58 \\
Chr7 & 21 & 23 & 0 & 4 & 48 \\
Chr8 & 22 & 30 & 1 & 1 & 54 \\
Chr9 & 13 & 33 & 0 & 2 & 48 \\
Chr10 & 11 & 19 & 0 & 1 & 31 \\
Chr11 & 19 & 15 & 0 & 1 & 35 \\
Chr12 & 13 & 28 & 1 & 2 & 44 \\
Chr13 & 19 & 13 & 0 & 0 & 32 \\
Chr14 & 24 & 13 & 0 & 1 & 38 \\
Chr15 & 11 & 16 & 2 & 1 & 30 \\
Chr16 & 12 & 20 & 1 & 0 & 33 \\
others & 299 & 102 & 17 & 15 & 433 \\
Total & 703 & 581 & 32 & 47 & 1363 \\
\hline
\end{tabular}

2 


\section{Table 2 (on next page)}

Table 2 SNP markers located within IncRNA regions and significantly associated with the composition of fatty acid in oil palm mesocarp

SNP markers located within IncRNA regions and significantly associated with the composition of fatty acid in oil palm mesocarp 
Table 2 SNP markers located within IncRNA regions and significantly associated with the composition of fatty acid in oil palm mesocarp

\begin{tabular}{|c|c|c|c|c|c|c|c|c|c|}
\hline IncRNA & \multicolumn{3}{|c|}{ SNP position } & \multirow{2}{*}{$\frac{\text { Related traits }}{\text { palmic acid content }(\mathrm{C} 16: 0)}$} & \multirow{2}{*}{$\frac{\text { P-Value }}{0.000794231}$} & \multicolumn{3}{|c|}{ Nucleotide types } & \multirow{2}{*}{$\frac{\text { Ave_Het }}{0.2301}$} \\
\hline MSTRG.2123 & Chr1 & 26348240 & Intron & & & K & G & $\mathrm{T}$ & \\
\hline MSTRG.2168 & Chr1 & 27359487 & Intron & Stearic Acid content (C18:0) & 0.000671054 & M & C & $A$ & 0.318 \\
\hline MSTRG.2168 & Chr1 & 27366210 & Exon & Stearic Acid content (C18:0) & 0.000667117 & $\mathrm{R}$ & G & $A$ & 0.2188 \\
\hline MSTRG.4816 & Chr2 & 10805479 & Intron & total oil content & 0.000916663 & $A$ & G & $\mathrm{R}$ & 0.4067 \\
\hline MSTRG.7396 & Chr2 & 56802941 & Intron & palmic acid content (C16:0) & 0.00038596 & G & $\mathrm{R}$ & $A$ & 0.1968 \\
\hline MSTRG.7396 & Chr2 & 56803122 & Intron & palmic acid content (C16:0) & 0.000874185 & $\mathrm{~T}$ & Y & $\mathrm{C}$ & 0.208 \\
\hline MSTRG.7396 & Chr2 & 56767445 & Exon & total oil content & 0.000732102 & G & $\mathrm{R}$ & $A$ & 0.1425 \\
\hline MSTRG.7397 & Chr2 & 56767445 & Intron & total oil content & 0.000732102 & A & $\mathrm{R}$ & G & 0.2518 \\
\hline MSTRG.10380 & Chr3 & 37185131 & Intron & linoleic acid content (C18:2) & $1.49 \mathrm{E}-09$ & A & G & $\mathrm{R}$ & 0.375 \\
\hline MSTRG.11378 & Chr4 & 4103682 & Intron & total oil content & 0.000223078 & $\mathrm{~T}$ & W & $A$ & 0.1605 \\
\hline MSTRG.11378 & Chr4 & 4103761 & Intron & total oil content & 0.000414367 & $\mathrm{~T}$ & $\mathrm{Y}$ & C & 0.1565 \\
\hline MSTRG.13525 & Chr4 & 50679685 & Intron & total oil content & 0.000982483 & G & A & $\mathrm{R}$ & 0.4258 \\
\hline MSTRG.15295 & Chr5 & 25374494 & Exon & linoleic acid content (C18:2) & 0.000862608 & $A$ & $\mathrm{R}$ & G & 0.4562 \\
\hline MSTRG.15330 & Chr5 & 27273554 & Intron & total oil content & 0.000838241 & C & $\mathrm{T}$ & $\mathrm{Y}$ & 0.3712 \\
\hline MSTRG.16341 & Chr5 & 46800615 & Intron & palmic acid content (C16:0) & 0.000916155 & Y & C & $\mathrm{T}$ & 0.495 \\
\hline MSTRG.16341 & Chr5 & 46773860 & Intron & linoleic acid content (C18:2) & 0.000914323 & G & A & $\mathrm{R}$ & 0.375 \\
\hline MSTRG.16342 & Chr5 & 46773860 & Intron & linoleic acid content (C18:2) & 0.000914323 & G & A & $\mathrm{R}$ & 0.3871 \\
\hline MSTRG.16344 & Chr5 & 46800615 & Intron & palmic acid content (C16:0) & 0.000916155 & Y & C & $\mathrm{T}$ & 0.495 \\
\hline MSTRG.16345 & Chr5 & 46800615 & Intron & palmic acid content (C16:0) & 0.000916155 & G & $\mathrm{R}$ & & 0.3432 \\
\hline MSTRG.17117 & Chr6 & 5087561 & Intron & total oil content & 0.000433608 & $\mathrm{~T}$ & Y & $\mathrm{C}$ & 0.4061 \\
\hline MSTRG.17118 & Chr6 & 5087561 & Intron & total oil content & 0.000433608 & G & $\mathrm{R}$ & A & 0.4283 \\
\hline MSTRG.17644 & Chr6 & 21539684 & Intron & total oil content & 0.000343467 & C & $\mathrm{T}$ & $\mathrm{Y}$ & 0.4793 \\
\hline MSTRG.17644 & Chr6 & 21539697 & Intron & total oil content & 0.000776114 & $\mathrm{~T}$ & C & $\mathrm{Y}$ & 0.4851 \\
\hline MSTRG.28271 & Chr11 & 28838549 & Intron & total oil content & 0.000973214 & A & G & $\mathrm{R}$ & 0.4968 \\
\hline MSTRG.28993 & Chr12 & 15680141 & Intron & linoleic acid content (C18:2) & 0.000661354 & Y & $\mathrm{T}$ & $\mathrm{C}$ & 0.475 \\
\hline MSTRG.28993 & Chr12 & 15680161 & Intron & linoleic acid content (C18:2) & 0.000820421 & $\mathrm{R}$ & G & $A$ & 0.4729 \\
\hline MSTRG.29406 & Chr12 & 21510854 & Intron & Stearic Acid content (C18:0) & 0.000778935 & G & $\mathrm{R}$ & A & 0.1147 \\
\hline
\end{tabular}


